\title{
ENTREVISTA AL DOCTOR PEDRO SALAZAR UGARTE ${ }^{1}$
}

\section{ABRIL USCANGA BARRADAS}

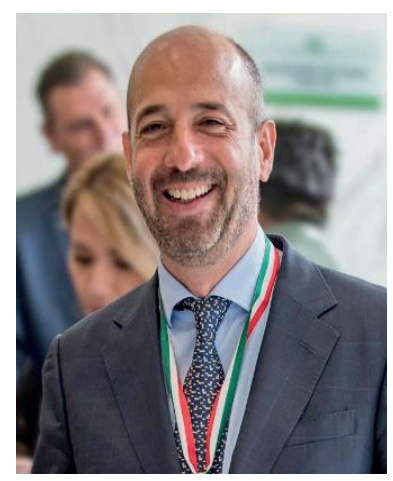

Licenciado en Derecho y Doctor en Filosofía Política. Es Director del Instituto de Investigaciones Jurídicas de la UNAM e investigador "B" de Tiempo Completo del mismo. Miembro del Sistema Nacional de Investigadores (Nivel III). Integrante del Grupo Nacional Mexicano de la Corte Permanente de Arbitraje de la Haya. Forma parte del Claustro de Doctores de la Facultad de Derecho de la UNAM y es profesor de teoría política en la misma.

Entre sus libros se encuentran: El Poder Ejecutivo en la Constitución mexicana. Del metaconstitucionalismo a la constelación de autonomías (FCE, 2017), Estado laico en un país religioso. Encuesta Nacional de Religión, Secularización y Laicidad (en coautoría con Paulina Barrera y Saúl Espino. IIJ-UNAM, 2015); La República Laica y sus libertades. Las reformas a los artículos 24 y 40 constitucionales. (IIJ-UNAM 2015); Derechos Humanos y Restricciones. Los dilemas de la justicia (en coautoría con Luis Daniel Vázquez y Alejandra Medina Mora. Porrúa, 2015. ); Derecho y Poder. Derechos y garantías (Fontamara, 2013);

${ }^{1}$ Este documento constituye una transcripción de la entrevista realizada. Se ha revisado sin alterar el estilo coloquial propio de una conversación, considerando que esta característica enriquecería el texto que se presenta al lector. La entrevista fue realizada con la colaboración de Andrea Valencia Bermúdez y Salvador Villaseñor Olguín. 
Crítica de la mano Dura. Cómo enfrentar la violencia y preservar nuestras libertades (Océano 2012); La Reforma Constitucional de Derechos Humanos. Un nuevo paradigma (coordinado con Miguel Carbonell en 2012); La Democracia Constitucional. Una radiografía teórica (FCEUNAM 2006); El Derecho a la libertad de expresión frente al derecho a la no discriminación (en coautoría con Rodrigo Gutiérrez; CONAPRED-UNAM 2003); La mecánica del Cambio Político (en coautoría con Ricardo Becerra y José Woldenberg. Cal y Arena, 2000).

\section{ENTREVISTA AL DOCTOR PEDRO SALAZAR UGARTE}

Abril Uscanga Barradas: Me encuentro con el Doctor Pedro Salazar Ugarte para entrevistarlo. Le agradecemos muchísimo que nos haya ofrecido de su tiempo para realizar esta entrevista.

Pedro Salazar Ugarte: Al contrario Abril, muchas gracias por abrirme este espacio para a través de las páginas de su Revista poder comunicar algunas impresiones con los alumnos de nuestro posgrado en derecho.

AUB: Nuevamente gracias, doctor. Quisiera iniciar preguntándole un poco acerca de su trayectoria, especialmente los inicios de esta y sus estudios, nos gustaría saber en particular, ¿cuáles fueron sus motivaciones para estudiar derecho?

PSU: Claro. Yo decido estudiar derecho en los últimos años de preparatoria, en realidad mi padre había estudiado derecho, era abogado de la libre de derecho, quien ejerció más bien en la práctica privada y comercial, pero tenía esa formación y también mis dos hermanos mayores habían estudiado derecho, ninguno de ellos ejerció la práctica propiamente profesional, pero su formación base fue jurídica y, en ese sentido, eran figuras que me habían resultado ejemplares en mi vida y era una 
carrera que me era cercana; siempre me gustó mucho la argumentación, la deliberación y me agradaba todo lo que tenía que ver con la comunicación oral, y en ese sentido, el derecho siempre me ha parecido una carrera sumamente atractiva y no me arrepiento, aunque debo decir que una vez que entré a estudiar, las ramas que más me llamaron la atención, y en las que me especialicé, son las que tenían que ver con cuestiones más conceptuales, más teóricas, más hacia conceptos y teorías del derecho y, no necesariamente hacia alguna de las ramas enfocadas a la práctica o el litigio.

AUB: Considero que en gran medida es enriquecedor conocer parte de la formación de los académicos y de quienes llegan a marcar sus vidas, ya que nos hace penetrar un poco más en ese ámbito personal y, en ese mismo sentido, nos gustaría conocer, si nos permite, ¿cuáles fueron aquellos profesores, probablemente incluso autores, de algunos libros que hayan marcado su experiencia en los inicios de su formación académica?

PSU: Con mucho gusto. Desde el punto de vista de los profesores, considero que mi formación está marcada, durante mis estudios de licenciatura, por la identidad intelectual de tres grandes maestros: sin duda por el maestro Carlos de la Isla, un filósofo moral muy riguroso en su pensamiento, un hombre liberal y muy comprometido con las causas más emancipadoras del ser humano. Otro maestro fundamental fue Víctor José Blanco Fornieles, teórico del derecho Kelseniano muy sólido en su formación y sobre todo muy riguroso en su razonamiento, un hombre formado en lógica formal y al mismo tiempo en argumentación jurídica, que influyó mucho, no solamente en mi formación conceptual y teórica, sino también en mi formación personal, porque al igual que el profesor Carlos de la Isla Veraza, fue un gran modelo de persona íntegra y 
comprometida con causas en las que yo también creo. Finalmente, otro filósofo en la carrera, Rodolfo Darío Vázquez Cardozo, un autor muy conocido dentro y fuera de México, muy cercano con quien igualmente me honra conservar hasta el día de hoy una relación de amistad y que ha dedicado su obra al pensamiento liberal, un pensamiento liberal igualitario en el que yo adscribo mi propia identidad intelectual. Sin duda son los tres grandes maestros de mi formación jurídica. Hay otras figuras que van a incidir mucho en mi formación intelectual: sin duda Isaac José Woldenberg Karakowsky en el mundo profesional y en mis primeros años de práctica como asesor jurídico en el Instituto Federal Electoral, quien además se convirtió en un amigo, un referente, modelo intelectual comprometido con su tiempo y al mismo tiempo de persona honorable, capaz y absolutamente ejemplar; también mencionaré a mi tutor de doctorado, con quien tengo una relación muy cercana, casi familiar, me refiero a Michelangelo Bovero, profesor de la escuela de Turín, alumno de Norberto Bobbio, quien fue absoluta y totalmente generoso en el compartir conmigo sus conocimientos. Y también hay mujeres que inciden de manera relevante en mi formación, sobre todo en aquellas que me llevan a conocer y a reflexionar desde la perspectiva crítica del pensamiento feminista, como es el caso de Marta Lamas Encabo, con la que particularmente también tengo una relación de amistad y me hace mirar las cosas desde una perspectiva distinta a la que tradicionalmente yo había aprendido.

Respecto de autores, soy un lector, desde el inicio de mis estudios, de autores clásicos, de pensamiento liberal, como lo es Immanuel Kant y John Locke, o desde una perspectiva más contemporánea puedo mencionar a Hans Kelsen, un autor que me complace su lectura; posteriormente Luigi Ferrajoli se va a convertir en un referente intelectual en la 
bibliografía, pero también en la dinámica y en la relación personal. Adicionalmente, fuera de la teoría jurídica, me interesan lecturas de teoría política, que de hecho es mi otra formación, y ahí sin duda, Norberto Bobbio se vuelve un referente intelectual internacional muy importante para mi formación. Y finalmente, para mencionar autores mexicanos, me referiré a Luis Salazar Carrión, un filósofo de la política de estirpe Bobbiana pero con pensamiento propio que tiene además una formación de base marxista, lo que lo hace muy interesante en su línea de pensamiento. Por mencionar algunos.

AUB: Muchísimas gracias, doctor. Por lo que nos comenta, tiene una fuerte influencia teórica y filosófica. En algún punto, esto lo llevó a interesarse por la investigación y, se dio la oportunidad de ser investigador en el Instituto de Investigaciones Jurídicas. ¿Cuándo sucedió esto y quién lo invita en principio?, ¿quién lo formó aquí en el Instituto?

PSU: Eso es muy interesante, porque en efecto hay un momento cuando decido estudiar en el doctorado en el que opto por la vida académica y además opto con mucho compromiso y mucho agrado y, no me he arrepentido de esa decisión. Yo creo que hay distintas rutas en las cuales un profesionista o una profesionista del derecho puede orientar su formación: obviamente una de las primeras elecciones implica el ser abogados postulantes, a lo cual yo no me dediqué ni siquiera a los inicios de mi carrera; la siguiente alternativa, entre otras, es el sector público, en el cual sí tuve oportunidad de trabajar, sobre todo en el Instituto Federal Electoral en ese entonces denominado así, como asesor jurídico, donde tuve la experiencia más cercana a la práctica del derecho, siendo una experiencia muy enriquecedora; otra alternativa, la iniciativa privada, entendida como un espacio muy legítimo de dedicación profesional al cual no me interesó dedicarme; también se debe mencionar que la judicatura 
es donde a veces los académicos incursionan de ida y vuelta en distintos aspectos de la vida judicial, yo hasta el día de hoy no he tenido oportunidad, ni interés en ello. Yo encontré la vía académica y fue esta la que me pareció muy atractiva. Quizá, el primer momento en el que decido dónde y con quién estudiar, es cuando escucho a Michelangelo Bovero en una conferencia en el Instituto Federal Electoral. Todo en la vida te va orientando y recuerdo haber dicho "yo quiero estudiar con esta persona”, a partir de ahí me lo propuse y se fue abriendo la posibilidad, hasta que se materializó en una realidad.

Viví varios años en Europa, en Italia, en España, en Oxford donde tuve una experiencia de varios años de formación, y cuando volví a México mis disyuntivas eran entre la universidad privada, como la universidad en la que yo había estudiado, el ITAM, o la universidad pública pero con una connotación distinta a los centros de investigación públicos como era el CIDE, en donde también tenía alguna posibilidad de incursionar, pero para mí, la máxima aspiración era Instituto de Investigaciones Jurídicas, aunque lo veía muy lejano, dentro otras cosas por no ser egresado de nuestra Facultad de Derecho. Fue Hugo Alejandro Concha Cantú durante una cena en casa de Jesús Silva-Herzog Márquez quien me dijo, en un periodo en el que yo todavía no terminaba el doctorado, que él era Secretario Académico en Jurídicas con el Doctor Diego Valadés, quien era el Director en ese entonces del Instituto, y que le gustaría proponer mi currículum, para que eventualmente pudiera ingresar al Instituto. En ese entonces tenía amigos entrañables, como Lorenzo Córdoba Vianello, Rodrigo Gutiérrez Rivas, Miguel Carbonell, Oscar Cruz Barney, que ya se encontraban en el IIJ. A mí me parecía ideal entrar al Instituto y al final se materializó esa posibilidad, cosa que me dio mucho gusto y que me permitió además empezar mi carrera académica desde la UNAM en 
la investigación, pero también en la docencia, la cual sigo manteniendo vigente hasta el día de hoy.

AUB: Doctor, ha comentado algo que sin duda causa expectativa dentro de la Universidad, me refiero a las formaciones endogámicas de las Universidades, que aunque son útiles, no deben descartar la riqueza que amerita el que existan formaciones externas, esto seguramente fue muy benéfico para el Instituto. Ahora que usted es director podría comentarnos, ¿qué representa para usted la UNAM?

PSU: En verdad a mí me gusta decir que soy un caso de expropiación voluntaria exitosa, porque disfruto enormemente la vida universitaria; tengo ya más de tres lustros en la UNAM y digo con convicción que soy unamita por decisión propia en una etapa adulta. Yo estudié cuatro años en el ITAM y en la UNAM llevo tres lustros, sí hay una diferencia relevante. Creo que esa doble experiencia para mí en lo individual ha sido sumamente enriquecedora y me ha llevado a valorar las bondades de ambos perfiles y de ambas orientaciones, pero sobre todo a ponderar de manera muy positiva el valor de la universidad pública y el sentido incluyente, el sentido dinámico de una universidad que tiene las particularidades de serlo como la UNAM. Me ha llevado también a una valoración muy positiva de que la universidad sea un espacio de recreación de la diversidad en los sentidos más amplios y que al mismo tiempo esta sea una de sus cartas de identidad. A mí me gusta decir que yo, por supuesto, soy de la Facultad de Derecho y lo soy de muchas maneras: lo soy como conferenciante, que ahora como autoridad asisto en muchas ocasiones a actos protocolarios; pero lo que más me importa y lo que más me gusta, es ser maestro, un maestro muy dedicado a sus aulas y a la formación de sus alumnas y alumnos de los cuales me siento muy orgulloso. Me gusta ser profesor, lo he sido en muchas instituciones dentro y fuera del 
país, además, en donde enseño los conocimientos teóricos que aprendí en mi formación básica, de doctorado y en ambas dimensiones, desde la perspectiva jurídica y desde la perspectiva política, que son mis dos formaciones, y en ese sentido también soy un poco ortodoxo en mi formación, es decir, a mí me parece que enriquece mirar los temas desde distintas perspectivas al igual que enriquece una trayectoria que tiene distintos espacios de experiencia. Para mí el reto más interesante fue cuando decidí que a pesar de no haber estudiado en nuestra universidad, pero sintiéndome totalmente parte de ella, quería dirigir al Instituto de Investigaciones Jurídicas de la UNAM y cuando me lo propuse y tuve la posibilidad de hacerlo, lo asumí con una enorme responsabilidad y con un absoluto sentido de pertenencia. Y en aquellos contextos me preguntaban si consideraba que no haber estudiado en la UNAM era un problema y yo decía, y lo sigo diciendo, que al contrario, que yo creo que es una riqueza que te permite valorar la diversidad desde una perspectiva fresca, distinta y sumamente comprometida.

AUB: Doctor, no puedo estar más de acuerdo con usted, en la tómbola de la vida a veces no se pueden elegir, ni tomar todas las decisiones, pero las que se toman consciente y racionalmente a veces son las que más proyectan nuestra vida, como es su caso. Creo también que la Universidad, acoge, representa y tiene que unir todas las visiones, todos los proyectos de vida, incluso de los que no nacen en la Ciudad de México, todas las personas que representa este país.

PSU: Por supuesto. Si la Universidad pretende ser, como de hecho lo es, la Universidad de la Nación debe de estar abierta a cualquier origen formativo. Yo estudié con los maristas en el Colegio México, después Instituto México y después en el Centro Universitario México, el CUM. Posteriormente estudié cuatro años en una universidad privada, aunque 
después estudié el doctorado en una universidad pública europea, en la que obtuve muy valiosa formación. Y ulteriormente he dedicado mi vida profesional, ya como académico, totalmente a la universidad pública nacional y creo que esa apertura a los distintos orígenes, como puede ser el mío, tiene mucho que ver con lo que usted señala, Abril: que todas las jóvenes y todos los jóvenes por ubicarse geográficamente, totalmente fuera de la universidad, desarrollan sus estudios de licenciatura, incluso a veces de posgrado, en universidades públicas o privadas en otras entidades federativas y que vienen a enriquecer los claustros académicos cuando se incorporan. Nosotros tenemos investigadores muy interesantes, le menciono por ejemplo, el caso de la doctora María de Jesús Medina Arellano, ella creció y estudió en Nayarit su licenciatura y, la universidad de Nayarit creó un programa especial para tener por primera vez egresados suyos que contaran con un doctorado en el extranjero. Realizó estudios en la Universidad de Edimburgo, relacionados con bioética y, en México realizó -muy joven todavía ella- una estancia en el Instituto de Investigaciones Jurídicas en un proyecto que se llama verano de la investigación jurídica que está orientado a jóvenes. Ella terminó sus estudios de doctorado y a través del programa de renovación de la planta académica SIJA, que se abre desde la Rectoría del Dr. José Ramón Narro Robles, fue contratada cuando aún no llegaba a tener treinta años y ahora es una investigadora joven pero ya consolidada que ha logrado además abrir metas de investigación en materia de bioética muy relevantes a la universidad.

Bueno, ahí tiene un ejemplo de alguien que no tuvo la oportunidad de estudiar en nuestra Facultad de Derecho, aquí en Ciudad Universitaria, tampoco en alguna sede de la UNAM, pero que es sin duda una historia de éxito en la academia y en la investigación. 
AUB: Así es, de hecho conozco diferentes casos de colegas que no teniendo la oportunidad de integrarse en un inicio por su localización geográfica o diversas circunstancias, finalmente eligen apasionadamente incorporarse, ya sea en estudios posteriores o a través de la labor académica. Lo digo también como experiencia personal, ya que soy de Veracruz, entonces entiendo perfectamente, y la verdad es que creo que para nosotros es una gran oportunidad incorporarnos en esta universidad, incluso cuando las condiciones de vida en principio no las podamos decidir.

PSU: Sin duda.

AUB: Doctor, pasando a temas relacionados a su gestión como director del Instituto de Investigaciones Jurídicas, y siempre me gusta comentar, a mi parecer es el Instituto más importante que tenemos en el país y de donde sale la mayor parte o casi la totalidad de la investigación más relevante de punta o de frontera que tenemos. ¿Cuál considera usted en particular que fueron los retos que encontró? Como sucede en cada administración, por supuesto, hay retos que afrontar, y también desde la otra perspectiva, ¿cuáles han sido los logros que se han alcanzado?

PSU: Yo creo, y en verdad le digo, coyunturalmente soy director y dejaré de serlo, entonces hablo con la subjetividad de quien dirige pero con objetividad de quien puede tomar distancia. Yo creo que el Instituto de Investigaciones Jurídicas es el centro de investigación jurídica más relevante de Iberoamérica y es un referente internacional.

Cuando me preguntan de retos, comento qué pretendo hacer en los próximos años junto con mi comunidad, la que lleva el liderazgo y la que lo hace posible, convertirnos en el referente mundial de cómo hacer investigación jurídica. Tenemos hoy una vinculación internacional muy relevante en América Latina, en general en Sudamérica, en sentido amplio 
por supuesto con España desde hace mucho tiempo, pero en los últimos años, y ese ha sido uno de los retos, que no inició conmigo pero que me ha tocado consolidar, la de diversificar nuestros puntos de vinculación internacional. Hoy jurídicas trabaja estrechamente con universidades de Reino Unido, Alemania, Francia e Italia, y uno de los retos, desde la gestión anterior del doctor Héctor Fix Fierro, pero con mucho ahínco en la mía, es la diversificación hacia Asia Pacífico, en donde tenemos cada vez más vinculación, por ejemplo, con universidades de China, Japón, la India, Corea del Sur y Singapur. Hemos diversificado hacia allá también nuestra agenda de investigación y por supuesto hacia los Estados Unidos, cada vez con más fuerza. Tenemos además investigadoras e investigadores, pero sobre todo investigadoras que tienen formación de posgrado en universidades norteamericanas y que nos han ayudado a mantener una relación muy estrecha con las Universidades de Stanford, de Princeton, de Harvard, de Yale y con otras instituciones del sur de Estados Unidos o el norte de nuestro país. Encontramos ahí un reto de posicionamiento que tiene que ver con lo más importante que hacemos, que es generar conocimiento a propósito del Derecho.

El otro gran reto que sigue abierto es el de lograr dotar de una identidad intelectual al tipo de investigación jurídica que llevamos a cabo. También que nuestro claustro -y esto no inició conmigo pero conmigo se ha consolidado- sea cada vez más interdisciplinario. En el Instituto de Investigaciones Jurídicas hay juristas duros, juristas duras, pero hay también quienes como yo tenemos formaciones híbridas: somos muchos los que tenemos formación jurídica base y posgrado en otra disciplina, ya sea sociología, ciencia política, demografía, economía o, como en mi caso, de teoría política o viceversa, también quienes tienen una formación de base en otra carrera y tienen posgrado, doctorado en derecho. 
Esos "híbridos" han hecho que problematicemos los temas jurídicos desde perspectivas distintas a las internas del derecho y también, tenemos investigadores que tienen formación netamente periférica o externa al derecho como lo son demógrafos, sociólogos, politólogos etc., y ese claustro es uno que delibera y dialoga mucho en torno a su propia identidad y quehacer intelectual y creo que ahí hay un reto muy grande; por ese reto es por el que creo que nos podemos volver en el referente mundial de cómo hacer investigación jurídica, porque lo que estudiamos son temas de relevancia jurídica con una perspectiva integral y creo que ahí se explica también el gran impacto de nuestras investigaciones, para que impacten en la realidad nacional y en la realidad mundial, y lo hacemos de muchas maneras, con una política editorial muy cuidadosa en su calidad pero muy ambiciosa en su impacto, como un proyecto que surge en la gestión anterior de esta administración, que es un programa de proyectos académicos en el que traducimos en plataformas y medios de comunicación digitales mucho de lo que hacemos desde el punto de vista académico, para proyectarlo a través de las tecnologías con un gran impacto en muchas áreas. Hemos hecho por supuesto una muy ambiciosa propuesta de educación, de vinculación y de extensión académica para educación en los posgrados en los que podemos nosotros participar, muchos de ellos también en plataformas tecnológicas a través de diplomados, maestrías y cursos de distinta naturaleza.

Otro reto enorme: construir una comunidad diversa, plural, cruzada por varias generaciones, intereses y coordenadas ideológicas pero que al mismo tiempo tengan una coerción interna de un sentido de pertenencia común. Nuestras investigadoras más jóvenes tienen veintiocho años y nuestro investigador mayor tiene noventa y cinco, tenemos necesariamente distintas formaciones con distintas experiencias en un Instituto 
que está cumpliendo ochenta años, entonces hay un desafío de construcción de identidad que me ha tocado como director conducir en acompañamiento de mi comunidad.

Ha crecido la planta académica y se ha renovado, he ahí otro desafío enorme, porque las nuevas contrataciones son el futuro del Instituto y hay que lograr que se integren en una identidad compartida con quienes ya están acá. Un proyecto muy ambicioso, muy exitoso y oportuno. Jurídicas hoy cuenta con una Estación noroeste de investigación y docencia, en Tijuana, Baja California. Si bien las instalaciones físicas todavía no están operando, desde hace cinco años la agenda académica está absolutamente activa: tenemos once estudiantes de doctorado, hombres y mujeres, allá en Tijuana, realizamos una serie de congresos permanentes con el Colegio de la Frontera Norte, con la Universidad Autónoma de Baja California, generamos un consorcio universitario con la Universidad de Tucson en Arizona y con la Universidad de San Diego, realizamos seminarios de discusión permanente en torno a las tesis de nuestros doctorandos, hemos hecho actividades académicas diversas para incidir en temas de la frontera y hemos hecho una alianza estratégica con instituciones como el Centro Cultural de Tijuana (CECUT), el propio Colegio de la Frontera Norte (Colef), la Universidad Autónoma de Baja California (UABC). Es decir, Jurídicas tiene un proyecto académico muy pujante en Tijuana y una agenda en uno de los ámbitos más problemáticos del mundo contemporáneo. Y con esto concluiría: la investigación que hacemos nosotros tiene que ser técnicamente sólida e imparcial desde el punto de vista político y debe ser sobre todo socialmente útil, por lo mismo tenemos que estar en los grandes temas y problemas del mundo y del país. 
También quiero mencionar que tenemos por ejemplo un Observatorio del Sistema Interamericano de Derechos Humanos encabezado por Magdalena Cervantes Alcayde, el cual ha permitido que jueces y comisionados del Sistema Interamericano se reúnan en Jurídicas desde hace cinco años, una vez al año; tenemos un Observatorio muy relevante de Desaparición Forzada de Personas con la Universidad de Oxford, Minnesota y FLACSO México, que tiene la base de datos no oficial más completa de personas desaparecidas en el país; tenemos el SUDIMER que es el Seminario Universitario sobre Desplazamiento Interno, Migración, Exilio y Repatriación, el cual es un seminario con sede en Jurídicas que reúne a la red más potente de migrantólogas y migrantólogos, yo me atrevo a decir, no solo de México, sino de todo centro y Sudamérica; también tenemos el Seminario de Seguridad Ciudadana con sede en el Instituto, que es otro seminario universitario con particularidades de ese tipo, también todos estos a cargo de colegas mujeres y, tenemos también entre otras cosas, el Observatorio de Corrupción e Impunidad que también es un proyecto muy ambicioso y que está orientado a otro de los grandes problemas nacionales. Por otro lado está el Laboratorio Nacional de Diversidades a la cabeza de Alethia Fernández de la Reguera Ahedo, encargado del estudio de la riqueza como diversidad y no como problema en sus distintos ámbitos, desde el punto de vista de la discriminación, la violencia y los derechos humanos, por mencionar algunos elementos. Tenemos proyectos muy importantes sobre conocimiento jurídico y desarrollo cognitivo.

Tenemos otro claustro, del que me siento muy orgulloso porque es mérito final de esta gestión: es un claustro totalmente equilibrado en paridad de género donde en los ámbitos de la investigación, de los técnicos académicos, en el administrativo y en el del personal de base hay cin- 
cuenta/cincuenta. Aunque hasta ahora todos los directores hemos sido hombres, tenemos un cuerpo directivo con un liderazgo muy importante de mujeres. Dos de los brazos derechos de la dirección son muy destacadas funcionarias universitarias, una académica y otra administrativa. El cuerpo directivo está conformado en buena medida por un liderazgo femenino muy sólido que además ha venido reforzado con contrataciones equilibradas por mérito de mujeres jóvenes muy talentosas en los últimos años. Ese es otro de los grandes retos y de los grandes méritos de este Instituto.

AUB: Director es un gran placer escuchar esto que nos comenta. Sin duda los proyectos pueden generar una vinculación importante de México con el gobierno, puede generar nuevas perspectivas, incluso nuevas respuestas hacia los problemas que tiene el país y también considerando la agenda internacional, como usted lo ha comentado.

Quiero hacerle una pregunta respecto a lo que ha dicho con respecto a la investigación y la calidad de ésta. Como investigador, pero también como director conoce con mucho detalle cuales son los nuevos indicadores, cuáles son las nuevas necesidades en relación a la investigación, lo que se necesita actualmente, no solamente por parte del CONACyT y del Sistema Nacional de Investigadores, sino a nivel internacional. ¿qué representa una investigación de calidad actualmente?

PSU: Sin duda. Lo primero que hay que decir es que hay distintos ámbitos en los que se desarrolla la investigación académica. Cuando hablamos de investigación desde la UNAM no podemos omitir la investigación científica, que es importantísima, no podemos omitir que la investigación de ciencias sociales no es idéntica a la de humanidades, no es idéntica a lo que se hace en el ámbito de difusión cultural y no es idéntica a lo que se lleva a cabo en la multiplicidad, es muy variada, 
muy rica y muy robusta de institutos y de facultades en el ámbito de las ciencias llamadas duras, pero que son ciencias al igual que las otras y creo que cada una tiene parámetro de evaluación distinto. En algunos casos la investigación y su éxito se medirán por su impacto, por ejemplo, el desarrollo de una vacuna se medirá por la efectividad de la misma, en otras, se medirá por la generación de conocimiento para el conocimiento mismo, por su acumulación, por su superación o por la ruptura de verdades aparentemente comprobadas, es decir, la investigación es muy cruel con el conocimiento acumulado porque lo tiene que desmontar, lo tiene que desafiar y lo tiene que desmentir, si ese es el caso. Y en ese sentido la evolución científica y la medición del éxito de investigación va a depender del desarrollo de las investigaciones.

Por otro lado, en nuestro caso, nuestra investigación se mide en sus méritos dependiendo de los distintos tipos de investigación que llevas a cabo. La investigación filosófica es muy importante y tiene que ver entre otras cosas con las dimensiones axiológicas del derecho y del quehacer humano, se medirán por su capacidad de ir desarrollando paradigmas de pensamiento que se vuelvan referentes, que impacten e influyan en la reflexión conceptual teórica-filosófica de quien se dedica a este ámbito. Quienes se dedican a cuestiones vinculadas por ejemplo con el derecho a la no discriminación, hay una fortísima carga axiológica y una orientación más práctica del tipo de investigación que se lleva a cabo; quienes hacen derecho público de carácter constitucional, la investigación muchas veces se lleva a cabo en este instituto y desde su origen se medirá su fuerza, no solamente por su rigor, sino también por su capacidad de influir e incidir en las decisiones de política pública del gobierno; lo mismo sucede cuando estudiamos los temas migratorios en los cuales se espera aportar insumos en información que sean relevantes a la hora de la toma 
de decisiones públicas por parte de los encargados y las encargadas de las decisiones del Estado, es decir, no hay un solo parámetro para medir el éxito. Lo que sí, es que la investigación debe de ser muy rigurosa en su metodología, en su precisión y en su responsabilidad académica. Hay una cuestión muy importante en su honestidad e integridad académica y esos son parámetros valiosos para la investigación en todos lados, y en ese sentido, la investigación claro que es comparable en su calidad.

Ahora bien, hago una pregunta fundamental, ¿que se podría hacer para mejorar la calidad, capacidad e impacto de la investigación que se lleva cabo en México?

Por ejemplo, destinar más recursos. México no logró uno de sus compromisos en los sexenios anteriores que era siquiera llegar al uno por cierto del Producto Interno Bruto en inversión en materia de investigación. Países como Israel o como Corea del Sur han invertido entre el 4.5 y el 4.6 de su producto interno bruto en los últimos años y han tenido un éxito muy relevante, porque el dinero puesto en investigación no es gasto, es inversión. Creo que una de las cuestiones que deberíamos recordar, desde las universidades hacia gobernantes en turno, independientemente del partido político del que sea, es que, invertir dinero en ciencia tecnología y humanidades es invertir en el futuro del país, ahí hay un desafío que a veces olvidamos. Nunca hay que perder de vista que es muy difícil hacer investigación y que somos sumamente evaluados y sumamente exigentes con nosotros mismos en los circuitos académicos.

AUB: Estoy totalmente de acuerdo, Director. No quiero abusar con respecto al tiempo, me encantaría en alguna otra ocasión volver a conversar con usted pero quiero hacerle una última pregunta y pedirle un consejo, si es que me lo permite. En relación al primer punto, quien quisiera conocer un poco más acerca de Pedro Salazar el investigador, 
¿qué debería de leer?, y como segundo punto, ¿podría cerrar dándonos alguna recomendación para los nuevos investigadores?

PSU: Claro. Remarco que no estoy promocionando mi propia obra, pero si se quisiera leer qué hago y ver qué es lo que hago intelectualmente, yo recomendaría tres cosas: uno, el libro más teórico que tengo sobre cuestiones que me interesan de democracia y constitución que se llama "La democracia constitucional una radiografía teórica”, es del Fondo de Cultura Económica y es una versión de mi tesis de doctorado; en segundo lugar yo iría a buscar las cosas que he escrito sobre estado laico, porque creo que ahí se reúnen mis convicciones laicas pero también mi orientación ideológica, liberal e igualitaria y mi profunda objeción a todas las formas de discriminación; en tercer lugar me atrevería a buscar alguna crónica que haya escrito, sobre todo las que he escrito en la revista nexos, porque ahí encontrarán otra vertiente que me gusta mucho, que es la de explorar el trabajo, que si bien es académico, con más licencias, es el trabajo de la narrativa de crónicas, dentro de las cuales hay alguna que es la introducción de un libro, llamada "Crítica de la mano dura", en el cual creo que se refleja mi convicción a favor de los derecho humanos y mi objeción total al Estado autoritario y; finalmente, vería algunos programas que he tenido oportunidad de coordinar a lo largo de varios años en TVUNAM, porque ahí se pueden ver los intereses que tengo y, aunque no soy yo el que habla de los temas, sí se nota cuáles son de los de mi interés.

Y, por último, si alguien decide dedicarse a la investigación ¿qué les recomendaría yo?, en primer lugar, que definan muy bien y con mucha claridad por qué quieren dedicarse al trabajo intelectual, porque el trabajo intelectual es muy exigente, es muy demandante y muy celoso, hay que tener una convicción muy clara; en segundo lugar, que explore, 
pensando en el ámbito de ciencias sociales, específicamente de las jurídicas, su vocación para el estudio, a quien no le guste estudiar y no le guste leer en serio, no tiene nada que hacer en esta tarea; en tercer lugar, que decida con mucha claridad si se va a dedicar a la investigación o si lo que quiere simplemente es una formación profesional especializada, porque de esto dependerá mi recomendación a que estudie o no un doctorado. Para mí, doctores y doctoras, solo deben de ser quienes se dediquen a la investigación, ¿por qué?, porque es una inversión que requiere mucho tiempo, y solo se justifica si te vas a dedicar después al trabajo académico.

Después, dos cuestiones de consejo de apertura mental: cuestionar y romper sus propios prejuicios, ya que el que se dedica a este trabajo debe de estar combatiendo sus prejuicios todo el tiempo, porque estos son los principales enemigos del pensamiento libre; y la última, estar dispuesta o dispuesto con convicción a decir en todo lugar, siempre, lo que piensa, porque no hay nada peor que un académico que quiere agradar al auditorio, los buenos académicos dicen lo que con convicción piensan aunque estén equivocadas o equivocados y, de eso se trata la discusión y la deliberación, pero lo que no se vale, es cambiar el discurso dependiendo de quien se siente a escuchar, porque en ese momento pierdes la identidad y pierdes, desde mi punto de vista, la credibilidad intelectual.

AUB: Le agradezco mucho, Director, ha sido en verdad un gran placer el escucharlo, el estar aquí y convivir con usted. No quiero dejar la puerta cerrada, por favor acépteme más adelante otra invitación para hablar con nosotros. En este momento lo único que nos resta, es agradecerle a usted y a todos los que han podido hacer realidad esta entrevista. Doctor no es un adiós, es un hasta luego. 
PSU: Está totalmente invitada, además me da un gusto enorme que jovenes universitarias y universitarios sean quienes estén llevando a cabo este tipo de proyectos en la Revista del Posgrado en Derecho de la UNAM, así que bienvenidos también al Instituto, ya lo conocen, y la puerta de aquí está siempre abierta para todas y para todos, porque de eso se trata un Instituto de una universidad pública como esta.

AUB: Muchas gracias Director.

PSU: Gracias a ustedes. 